\title{
Evaluación de una estrategia de fidelización de clientes con dinámica de sistemas
}

\author{
Stefanía Peña Escobar \\ Gloria S. Ramírez Reyes** \\ Juan C. Osorio Gómez ${ }^{* * *}$
}

Recibido: 24/06/2013 - Aceptado: 12/12/2014

\begin{abstract}
Resumen
Los programas de fidelización de clientes son una herramienta de diferenciación y alto impacto para crear relaciones de largo plazo con los clientes de una empresa, de tal forma que se logre una mejora en cartera y recaudo, reducción de costos de atención a clientes, incremento en ingresos por mayores ventas a los clientes más rentables, ventas cruzadas, entre muchos otros beneficios. De acuerdo con esto, se presenta un modelo de dinámica de sistemas que permita analizar los beneficios económicos, en términos de rentabilidad, que se tendrían al implementar una estrategia de fidelización de clientes. Para ajustar y validar el modelo propuesto, se aplica en un caso real de una empresa comercializadora de productos agrícolas de la región. Los resultados de la simulación muestran que efectivamente el modelo cumple con su objetivo, y que el programa de fidelización logra importantes resultados en las finanzas de la organización.
\end{abstract}

Palabras clave: CLV (customer lifetime value), fidelización de clientes, dinámica de sistemas, marketing relacional, ROI (return on Investment).

Ingeniera Industrial, Universidad del Valle, Carrera 13 N. 5-22, industrial.buga@correounivalle.edu.co, $+(57)$ (2) 2370000 Ext. 201.

** MSc en Ingeniería Industrial, Universidad del Valle sede Buga, Cargo: Docente tiempo completo, Carrera 13 N.5-22, gloria.ramirez@correounivalle.edu.co,+(57)(2) 2370000 Ext. 201.

*** MSc en Ingeniería Industrial, Universidad del Valle, Cargo: Profesor titular, Ciudad Universitaria Meléndez Calle 13 No 100-00, juan.osorio@correounivalle.edu.co,+(57) (2) 3212167 Ext. 117. 


\section{Evaluation of a customer loyalty strategy with system dymamics}

\section{Abstract}

The customer loyalty programs are a tool of differentiation and high impact to create long term relationships with customers of a company, so as to achieve an improvement in the portfolio and collections, reduced costs of customer service, increased revenue from higher sales to the most profitable customers, cross selling, among many other benefits. Accordingly, we present a system dynamic model to analyze the economic benefits, in terms of profitability, which would be to implement a customer loyalty strategy. To adjust and validate the proposed model, its implementation is done in a real case of a marketer of agricultural products in the department of Valle del Cauca - Colombia. The simulation results show that indeed the model fulfills its purpose, and that the loyalty program achieved significant results in the finances of the organization.

Keywords: CLV (customer lifetime value), customer loyalty, system dynamics, relationship marketing, ROI (return on Investment). 


\section{INTRODUCCIÓN}

Actualmente, las empresas se ven enfrentadas a una gran competencia favorecida por la globalización de los mercados, por lo cual, se hace fundamental buscar la manera de diferenciarse de sus competidores; según [1], una empresa adquiere mayores oportunidades de éxito si evoluciona de una estructura basada en productos a una filosofía de marketing relacional, cuya base fundamental está constituida por el desarrollo de unas relaciones sólidas y mutuamente beneficiosas con los clientes, [2]; más allá de esto, se busca medir, gestionar y maximizar el valor del cliente al valorarlo como un activo de la organización mediante la implementación de estrategias que permitan desarrollar un oferta personalizada acorde con sus necesidades [3], y de esta manera obtener todos los beneficios asociados a la existencia de clientes fieles.

Sin embargo, desarrollar clientes fieles es un proceso que pone en evidencia la necesidad de diseñar e implementar estrategias coherentes con las políticas de cada organización orientadas a cumplir este objetivo, para lo cual, es necesario invertir esfuerzos y recursos, cada vez más escasos en la actualidad, lo que sitúa a la empresa con dos objetivos encontrados: 1) invertir recursos para fidelizar los clientes y obtener así, mayores beneficios económicos, 2) racionalizar el uso de los escasos recursos empresariales.

Se hará énfasis en la fidelización de clientes como estrategia capaz de generar importantes beneficios para una organización. En la literatura actual, autores como [4] y [5] coinciden en que los principales beneficios son: mejora en la cartera y recaudo, reducción de los costos de atención a los clientes, incremento en los ingresos por mayores ventas a los clientes más rentables, ventas cruzadas, entre muchos otros, aunque no se han encontrado investigaciones exhaustivas que permitan verificar y cuantificar efectivamente estos beneficios económicos. Actualmente, se identifica una carencia de herramientas que permitan analizar en términos cuantitativos los resultados de las estrategias de marketing implementadas por las empresas, y como resultados los directivos consideran las acciones de marketing como "costos" en el corto plazo, más que "inversiones a largo plazo" [6].

En este sentido, se hace necesario para los empresarios contar con herramientas que faciliten la toma de decisiones, y se pretende elaborar una propuesta de un modelo que permita analizar los beneficios económicos, en términos de la rentabilidad que se tendría al implementar una estrategia de fidelización de clientes, dadas las necesidades de diferenciarse en el mercado y al tiempo racionalizar el uso de los recursos empresariales.

Cabe resaltar que el propósito del presente estudio no es calcular datos exactos sobre los elementos financieros relacionados con la implementación de un programa de 
fidelización, sino más bien, estudiar el comportamiento del retorno sobre la inversión a través de la identificación y modelamiento de las relaciones entre las variables clave asociadas a la implementación de una estrategia de este tipo. Con esto en mente, se utiliza la dinámica de sistemas como herramienta para el estudio y diseño del modelo propuesto para determinar la factibilidad de la estrategia, en la medida que permite analizar los impactos, identificar las variables fundamentales a la hora de una implementación y evaluar las relaciones entre los distintos elementos asociados al programa de fidelización.

Los programas de fidelización de clientes son considerados "herramientas utilizadas para conocer mejor al cliente y conseguir vincularlo a la marca" [7]. La idea principal consiste en diseñar un programa eficiente de relación con los clientes, identificando los segmentos a los que va dirigido, el valor de dicho segmento y las acciones comerciales a las que son más sensibles. Gran parte del éxito de este tipo de programas consiste en desplegar todo un esquema de comunicación [8], de tal manera que el cliente conozca el programa y se sienta involucrado e importante para la compañía.

Reichheld, citado por [9], plantea que la satisfacción del cliente es una variable clave considerada en la formación de comportamientos de lealtad, y se define como el sentimiento del cliente de que el consumo aporta resultados placenteros frente a insatisfactorios, una comparación entre expectativas y resultados. De diversas investigaciones se tiene que la satisfacción, además de ser una condición necesaria, aunque no suficiente para que exista lealtad, es un buen indicador del valor percibido del cliente, el cual, al ser mayor permite incrementar el shareholder value [10].

Sobre fidelización de clientes vale la pena realizar un apunte respecto a la diferencia con el concepto de retención: fidelidad se considera como un constructo multidimensional más amplio y complejo que el de retención, ya que este último no es más que un indicador del primero [11], el cual está relacionado con el sentimiento de satisfacción del cliente con el producto o servicio, e impacta positivamente en el comportamiento del cliente [12]. La tabla 1 muestra una revisión de literatura sobre definiciones de programas de fidelización propuestos por diversos autores.

Tabla 1. Definiciones de programas de fidelización

\begin{tabular}{|l|l|}
\hline \multicolumn{1}{|c|}{ Autor } & \multicolumn{1}{c|}{ Definición } \\
\hline Rayner (1996) & $\begin{array}{l}\text { Un programa de fidelidad es un mecanismo para identificar y recompensar a clientes } \\
\text { leales. }\end{array}$ \\
\hline $\begin{array}{l}\text { Dowling y Uncles } \\
(1997)\end{array}$ & $\begin{array}{l}\text { Los programas de fidelidad son herramientas de marketing que tratan de reforzar la } \\
\text { fidelidad de los consumidores rentables, motivándoles a realizar nuevas compras a } \\
\text { través del incremento del valor global de la oferta. }\end{array}$ \\
\hline
\end{tabular}




\begin{tabular}{|l|l|}
\hline \multicolumn{1}{|c|}{ Autor } & \multicolumn{1}{c|}{ Definición } \\
\hline $\begin{array}{l}\text { Sharp y Sharp } \\
(1997)\end{array}$ & $\begin{array}{l}\text { Un programa de fidelidad es un esfuerzo estructural de la empresa para incrementar } \\
\text { el compromiso actitudinal y comportamental de los consumidores hacia sus ofertas de } \\
\text { mercado. }\end{array}$ \\
\hline $\begin{array}{l}\text { Benavent y Crié } \\
(1999)\end{array}$ & $\begin{array}{l}\text { Un programa de fidelización es un conjunto de acciones de marketing individualizado y } \\
\text { estructurado, organizado por una o varias empresas, para reclutar, identificar, mantener } \\
\text { y estimular a los mejores compradores de modo que aumenten su volumen de compra. }\end{array}$ \\
\hline $\begin{array}{l}\text { Benavent y otros } \\
(2000)\end{array}$ & $\begin{array}{l}\text { Un programa de lealtad es un conjunto de acciones dirigidas a estimular y mantener } \\
\text { clientes e incrementar la cantidad de producto comprada. }\end{array}$ \\
\hline $\begin{array}{l}\text { Bolton y otros } \\
(2000)\end{array}$ & $\begin{array}{l}\text { Los programas de fidelidad son mecanismos que pretenden establecer un alto nivel de } \\
\text { retención de clientes en los segmentos rentables aportándoles más satisfacción y valor. }\end{array}$ \\
\hline
\end{tabular}

Fuente: Adaptado de [11]

De las definiciones sobre programas de fidelización se puede llegar a un consenso donde en general, un programa de fidelización consiste en una herramienta que ofrece incentivos, permite identificar a los clientes más rentables y mantener su lealtad e incrementar las ventas (ver tabla 1. Definiciones de programas de fidelización).

La dinámica de sistemas corresponde a una herramienta para la construcción de modelos de simulación, con un enfoque diferente al utilizado tradicionalmente, el cual emplea datos empíricos como base para realizar cálculos estadísticos y así determinar el sentido y la correlación existente entre las diferentes variables que intervienen en el modelo; la evolución del mismo se realiza sobre la base de la evolución pasada de las variables independientes, y los parámetros de los sistemas de ecuaciones se extraen utilizando análisis estadístico.

De esta manera, puede decirse, que tradicionalmente se busca evaluar el comportamiento de un sistema, sin profundizar en el conocimiento de sus mecanismos internos. En contraste, se tiene que el objetivo básico de la dinámica de sistemas está orientado a comprender las causas estructurales que producen los comportamientos del sistema [13], y evaluar cómo diferentes acciones que se llevan a cabo sobre diversas partes del sistema acentúan o atenúan las tendencias implícitas en el comportamiento del modelo, a partir de un conocimiento más detallado sobre cada uno de los elementos que componen el sistema.

No pretende predecir detalladamente el futuro, sino, más bien, ser una herramienta para enriquecer el conocimiento del mundo real a través del análisis de sistemas complejos y poder evaluar la relación e impacto de las diferentes variables sobre el sistema que se esté evaluando; en términos prácticos, daría elementos de apoyo para el proceso de toma de decisiones. 
Posee un enfoque a largo plazo, es decir, un período de tiempo considerable que permita observar todos los aspectos significativos relacionados con la evolución del sistema a partir de las políticas establecidas [14], ya que, de nuevo, lo que se quiere evaluar son tendencias de comportamiento, pero esto solo es posible si desde el principio se han identificado claramente los causantes o motores del cambio en el sistema.

Tras un análisis cuidadoso de los elementos de un sistema cualquiera, es posible construir un modelo entendiendo la lógica interna del mismo, para poder realizar un análisis que lleve a un conocimiento de la evolución del sistema a largo plazo; es por esto que un ajuste a datos históricos ocupa un lugar secundario, si se compara con el análisis de la lógica interna y de las relaciones estructurales que componen el modelo.

Esta metodología permite:

- Identificar un problema

- Desarrollar hipótesis que apunten a encontrar la causa del problema

- Construir un modelo de simulación que permita analizar el comportamiento y la raíz del problema

- Verificar que el modelo reproduce satisfactoriamente el comportamiento real

- Probar en el modelo distintas alternativas que pueden dar solución al problema

Vensim es una herramienta visual de modelaje que permite conceptualizar, documentar, simular, analizar y optimizar modelos de dinámica de sistemas. Vensim provee una forma simple y flexible de construir modelos de simulación, sean lazos causales o diagramas de stock y de flujo, tal como lo propone la teoría referente a la dinámica de sistemas.

Mediante la conexión de palabras con flechas, se ingresan las relaciones entre las variables del sistema y se registran como conexiones causales. Con esto, el editor de ecuaciones facilita la finalización del modelo de simulación.

\section{METODOLOGÍA}

A continuación se presenta una descripción de la metodología utilizada para abordar el problema de investigación:

\subsection{Identificación de variables críticas}

De acuerdo con la revisión bibliográfica, se identifican las variables que se consideran más importantes para la construcción del diagrama de influencias o de relaciones. Estas variables se definen a continuación: 
Estímulo: se refiere en términos porcentuales al estímulo que recibe el cliente, a partir de una inversión en recompensas y medios de comunicación.

Ventas promedio por segmento de clientes: corresponde a las ventas promedio por cliente por período de tiempo, en cada uno de los segmentos de cliente; y que son afectadas por una tasa de crecimiento, según el nivel de estímulo que reciba el cliente.

Costo promedio por segmento de clientes: corresponde a los costos variables del programa de fidelización, se dividen por cada segmento de clientes y corresponden a una composición entre los costos asociados a la atención y los costos que se consideran como una inversión directa en el cliente, es decir, los descuentos y demás recompensas asociadas a la implementación del programa de fidelización.

Contribución marginal por segmento de clientes: corresponde a la utilidad que representa cada segmento de clientes, es decir, el sobrante en pesos que queda al descontar los costos variables de los ingresos totales (ventas), con lo cual se deben cubrir los costos fijos y queda una utilidad para la empresa.

Abandono por segmento de clientes: corresponde a la cantidad de clientes que abandona la relación con la empresa por unidad de tiempo; en este caso, es el flujo de salida de clientes.

Número de clientes por segmento: corresponde a la cantidad de clientes activos por unidad de tiempo en cada segmento de clientes.

Número total de clientes: corresponde a la cantidad total de clientes activos por unidad de tiempo, es decir, la suma de todos los clientes activos en cada uno de los segmentos de clientes.

Tasa de crecimiento ventas promedio por cada segmento de clientes: corresponde a un porcentaje al cual crecen las ventas promedio por cada segmento, a partir del estímulo recibido por los clientes de ese segmento.

CLV por segmento de clientes: corresponde al valor de vida en el tiempo de los clientes por cada segmento, y es una composición de la utilidad que representa el cliente (se tomará la contribución marginal en este caso) por un margen múltiple que es la combinación entre la tasa de descuento y una tasa de retención de clientes.

Customer equity: corresponde al valor total de la cartera de clientes, es decir, un promedio del CLV de los segmentos definidos por el número total de clientes en el sistema.

ROI: Se refiere al retorno sobre la inversión inicial realizada en la implementación del programa de fidelización. 


\subsection{Construcción del mapa de relaciones}

Con base en el proceso de identificación de variables críticas, se elabora el mapa de relaciones como una representación gráfica de la relación existente entre dichas variables. Para la construcción del mismo, es necesario tener en cuenta lo siguiente: teóricamente, se tiene que todos los clientes no responden de la misma manera a las acciones que ejecuta una organización para mantener y/o desarrollar una relación; así, resulta que algunos clientes realizan mayores compras con poco esfuerzo y representan un mayor valor para la empresa; por lo tanto, es razonable clasificar a los clientes en diferentes segmentos; se propone entonces aplicar los criterios de clasificación de inventarios A,B,C para agrupar los clientes por categoría o segmento, utilizando como criterio el monto de compra mensual, de manera que los clientes tipo A representan el $80 \%$ del total de ventas, los tipo B el $15 \%$ y los tipo C el $5 \%$ restante.

La idea base es la existencia de un programa de fidelización de clientes, el cual tiene asociado un costo promedio, compuesto por los costos de atención (plan de comunicación) y una inversión en el cliente (costos asociados a las recompensas); entre mayor sea el costo promedio, se proporciona un mayor estímulo al cliente, lo que, a su vez, puede tener dos efectos: un incremento en la tasa de abandono, por crear un efecto de saturación en el cliente al realizar demasiados contactos con el cliente en cada unidad de tiempo; al incrementar esta tasa, se aumenta el abandono de clientes del sistema y así el número de clientes en el segmento. El segundo efecto se considera como positivo, cuando tiene un impacto en el incremento de la tasa de crecimiento de ventas, es decir, que ante un mayor estímulo, siempre y cuando se encuentre en un rango aceptable, permite que las ventas crezcan a una tasa superior a la que tendría sin la implementación del programa de fidelización.

Ante este incremento en las ventas, se tiene un efecto positivo, en cuanto a la contribución marginal de clientes, que representa la utilidad de cada cliente; el efecto de compensación ante la contribución marginal son los costos promedio, ya mencionados. A su vez, la contribución marginal tiene un efecto positivo sobre el CLV (Customer Lifetime Value) de cada cliente, el cual se ve impactado positivamente por la tasa de retención de los clientes, y de manera negativa, por el crecimiento en la tasa de descuento con la cual se calcule el CLV.

Se tiene que una mejora en el CLV tiene un impacto positivo sobre el crecimiento del customer equity o valor de la cartera total de clientes, por lo cual esta variable se ve afectada también por la cantidad total de clientes. Con este valor entonces, se tiene el impacto positivo sobre el ROI o retorno sobre la inversión, puesto que a medida que el customer equity crezca a lo largo del tiempo, se obtiene un mejor rendimiento, teniendo en cuenta también la inversión realizada, puesto que ante un mayor valor de 
inversión, se tiene un menor retorno sobre la misma. Tal como se explicó al inicio de esta sección, la lógica referida anteriormente se repite en cada uno de los 3 segmentos de clientes definidos.

Finalmente es necesario tener en cuenta el flujo de clientes: se asume, que los clientes tipo A, al ser los mejores clientes, están satisfechos con el servicio, y son aquellos que van a hacer posible un efecto boca a boca positivo, mediante la interacción con una población de clientes; los clientes nuevos, al establecer una relación con la empresa, inician siendo clientes tipo $\mathrm{C}$, puesto que al no tener asociada una historia de compra, necesitan mayores costos de atención e inician la relación con montos pequeños en compras, y no representan grandes beneficios para la empresa en comparación con los beneficios que representan los clientes A; sin embargo, al realizar inversiones en el desarrollo de esta relación van pasando de un segmento de clientes a otro. Todo esto se aprecia gráficamente en la figura 1 (“diagrama de relaciones").

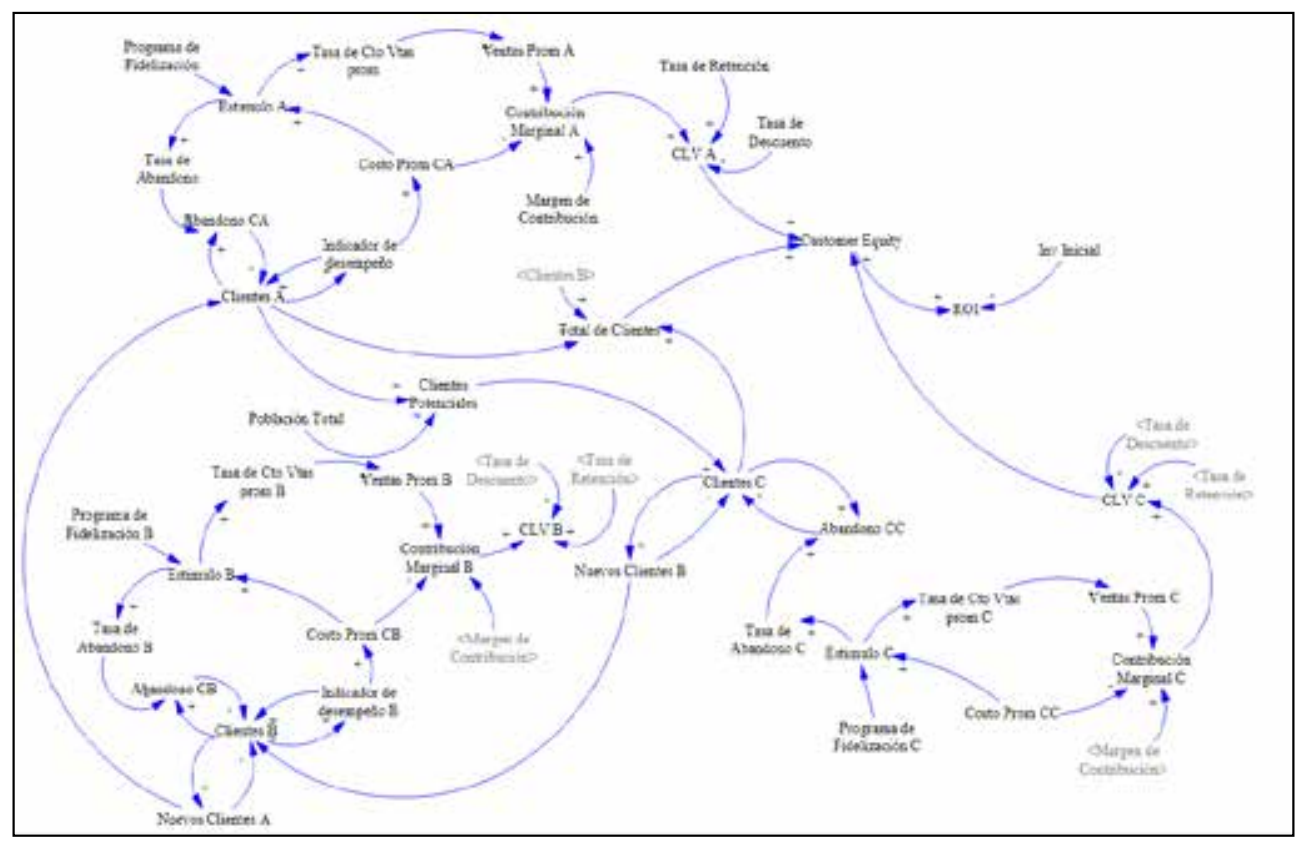

Figura 1. Diagrama de relaciones

Fuente: elaboración propia

\subsection{Construcción del diagrama de Forrester}

Se traslada el mapa de relaciones a un diagrama de Forrester (figura 2. "diagrama Forrester del modelo propuesto"); en este proceso se construye el modelo completo 
y es necesario incluir las variables auxiliares y ecuaciones que permitan realizar la simulación en el software de dinámica de sistemas que se vaya a utilizar; para este caso en particular se ha utilizado el Vensim ${ }^{\circledR}$.

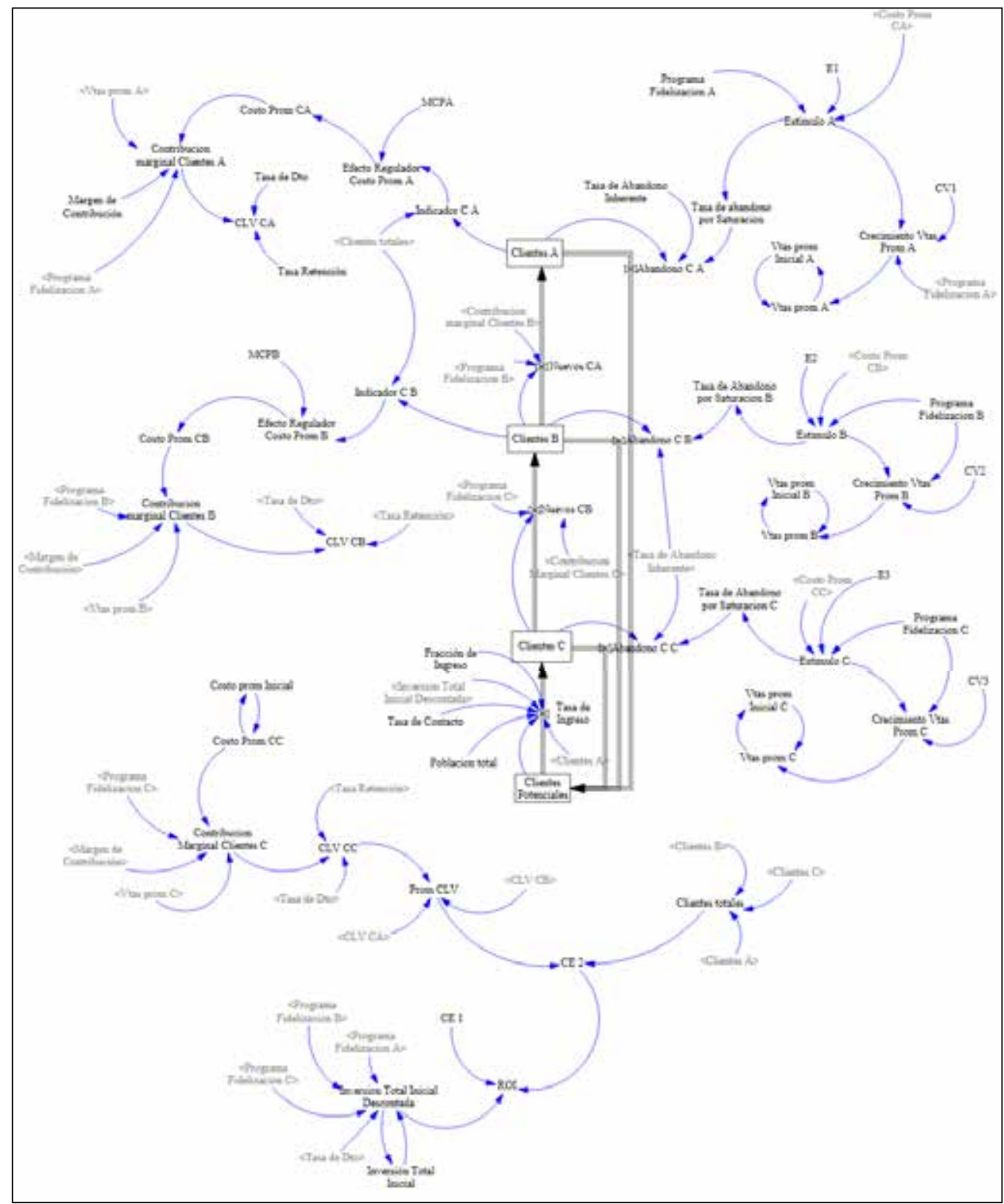

Figura 2. Diagrama forrester del modelo propuesto

Fuente: elaboración propia 


\subsection{Validación del modelo}

Para validar el modelo, es necesario tener datos reales que permitan ajustar los valores de los parámetros y evaluar que los resultados del modelo estén de acuerdo con la realidad. Para ello, se toman como referencia los datos reales correspondientes a una empresa Cooperativa de ganaderos ubicada en el Valle del Cauca-Colombia, específicamente en la actividad de comercialización de productos agropecuarios a los ganaderos.

Básicamente la información que se utiliza corresponde a todas las transacciones realizadas por los clientes en un período de cuatro años y medio, lo cual permite evaluar en un amplio período de tiempo el comportamiento de compra de los clientes.

Inicialmente se cuenta con una base de datos de 1.931 clientes para el año inicial, correspondiente a aquellos que realizan compras a crédito. Se plantea la necesidad de implementar un programa de fidelización por medio de una tarjeta inteligente para sus clientes, con el fin de potenciar el negocio, mantener y desarrollar la posición de la empresa en el mercado, acompañada de un "Club" al cual se le denominará "Club del Campo" como herramienta para la segmentación, vinculación y fidelización de clientes al proporcionarles asesoría y un apoyo directo en la ejecución de sus labores; además, por medio del club los clientes estarán unidos y podrán interactuar no solo por fidelidad a la marca, sino por intereses comunes, creando así vínculos más fuertes a través de la empresa promotora del club.

Con el fin de evaluar la coherencia en las relaciones establecidas durante la construcción del modelo y su capacidad para representar la realidad, se realizó la siguiente prueba: simular el modelo, sin programa de fidelización y evaluar el comportamiento de las variables número total de clientes y ventas promedio de los clientes $\mathrm{A}$, y confrontarlos con los datos reales. Los resultados se pueden apreciar en la tabla 2.

Tabla 2. Valor esperado y real en variables para prueba de ajuste

\begin{tabular}{|l|c|c|c|}
\hline \multicolumn{1}{|c|}{ Variable } & $\begin{array}{c}\text { Valor Inicial } \\
\text { (Realy simulación) }\end{array}$ & $\begin{array}{c}\text { Valor Final } \\
\text { Real }\end{array}$ & $\begin{array}{c}\text { Valor Final } \\
\text { Simulación }\end{array}$ \\
\hline Clientes Totales & 1.014 & 1.276 & 1.382 \\
\hline Clientes A & 121 & 180 & 142 \\
\hline Ventas promedio clientes A & $\$ 5.560 .965$ & $\$ 6.703 .333$ & $\$ 6.642 .700$ \\
\hline
\end{tabular}

Fuente: elaboración propia a partir de resultados obtenidos

Al comparar los valores finales se puede observar una gran coherencia entre los valores obtenidos con el modelo de simulación; interesa sobre todo el número total de clientes, puesto que en esta variable interviene un flujo de entrada a partir de la 
población objetivo, y los flujos de salida de clientes a partir de la tasa de abandono inherente, aparte del flujo de clientes entre un segmento y otro, del cual puede observarse una coherencia que se considera aceptable al evaluar el valor que adquiere la variable clientes A. (Ver tabla 2. Valor esperado y real en variables para prueba de ajuste).

\subsection{Experimentos de simulación, evaluación de escenarios y análisis de resultados}

Se ingresan los parámetros necesarios y se corre el modelo de simulación; posteriormente se realizan modificaciones al modelo y se corre nuevamente con el fin de evaluar la sensibilidad del mismo a ciertas variables y parámetros establecidos. A partir de las simulaciones del modelo original y los escenarios propuestos, se analiza el comportamiento de las variables de nivel, para determinar el impacto de las modificaciones propuestas. Estos resultados se presentan a continuación.

\section{RESULTADOS Y DISCUSIÓN}

La simulación en Vensim PLE se aplicó para un período total de 60 meses, ya que se considera un período razonable en el cual se pueden observar los efectos de una estrategia de marketing desarrollada por una empresa, tal y como lo es una estrategia de fidelización.

Para el análisis de los resultados como tal, se va a revisar de manera conjunta e individual el comportamiento de las variables clave, y a través de ellas, se evalúa el comportamiento del modelo propuesto.

Análisis: la cantidad de clientes totales para el periodo analizado tiene un comportamiento creciente de 1.276 clientes al iniciar el programa hasta llegar a un total de 1.777 clientes al final de la simulación, que equivale a un crecimiento del $39.2 \%$ respecto al valor inicial, y permite apoyar la idea de que la empresa está implementando diversas actividades de marketing orientadas a la captación de clientes; sin embargo, la idea es analizar el tema de la fidelización de los clientes actuales como uno de los resultados esperados del modelo; en este caso, se puede observar que los clientes A pasan de 180 a 239 clientes en el período de tiempo analizado, y representan un crecimiento del $32.7 \%$ respecto al valor inicial (ver tabla 3. "Valor inicial y final de las variables clientes totales y clientes por segmentos").

El modelo permite calcular el retorno sobre la inversión en el programa de fidelización teniendo en cuenta la inversión inicial para la implementación del programa y el cambio en el customer equity. La ecuación está construida con un condicional, de tal forma que si se requiere evaluar el comportamiento del modelo sin la existencia del programa de fidelización, pueda hacerse "inactivando" el programa para cada uno 
de los segmentos, caso en el cual no habría inversión inicial que medir; por lo tanto, el retorno sobre la inversión sería igual a 0 .

Tabla 3. Valor inicial y final de las variables clientes totales y clientes totales por segmentos

\begin{tabular}{|l|c|c|}
\hline \multicolumn{1}{|c|}{ Variable } & Valor Inicial (No de Clientes) & Valor Final (No de Clientes) \\
\hline Clientes Totales & 1.276 & 1.777 \\
\hline Clientes A & 180 & 239 \\
\hline Clientes B & 458 & 460 \\
\hline Clientes C & 638 & 1.078 \\
\hline
\end{tabular}

Fuente: elaboración propia a partir de resultados obtenidos

Al final de la simulación lo que interesa conocer es el retorno sobre la inversión, obtenido a partir de la implementación del programa de fidelización. Según la figura 3 puede evidenciarse que inicialmente el programa de fidelización aplicado a los 3 segmentos de clientes muestra un retorno negativo sobre la inversión, es decir, que con los beneficios que genera al principio no logra cubrir la inversión realizada en su implementación. Solo en el período 53, es decir, durante el quinto año de operación del programa se obtiene un retorno sobre la inversión igual a 0 , y adquiere una tendencia creciente, hasta llegar a un $22.1 \%$ para el período 59 , es decir, al finalizar el quinto año de operación.

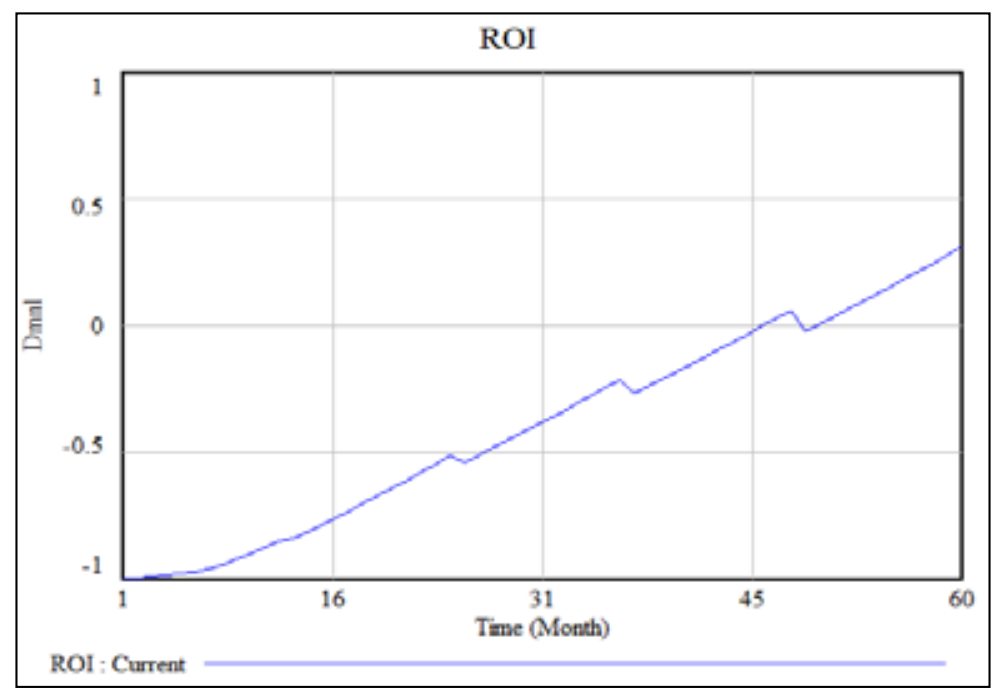

Figura 3. Comportamiento del ROI escenario base

Fuente: Reporte generado por el software Vensim ${ }^{\oplus}$ 
Este comportamiento puede ser explicado principalmente por varias situaciones:

- La inversión en la implementación del programa de fidelización es considerablemente alta.

- Los efectos positivos como el boca a boca (WOM), incremento en las ventas promedio por cliente, incremento en la cantidad de clientes y disminución de los costos de atención no se presentan de forma inmediata sino que tienen cierto retraso en el tiempo, mientras los clientes empiezan a responder a los estímulos generados por la empresa a través del programa.

- Los clientes tipo $\mathrm{C}$ son aquellos que presentan la menor contribución marginal $\mathrm{y}$, a su vez, son los clientes más numerosos, por lo tanto, incrementan de manera considerable los costos variables asociados al funcionamiento del programa sin incrementar de manera significativa sus compras promedio; por ello, inicialmente se tiene un flujo negativo (costo variable) que en los primeros períodos no se ve compensado por el incremento en los ingresos (ventas promedio).

- Para el caso de la inversión inicial, se tuvo en cuenta el valor del dinero en el tiempo, utilizando anualmente la misma tasa de descuento aplicada para el cálculo del CLV (12\%) lo cual proporciona una aproximación más realista para el cálculo del retorno sobre la inversión (ver figura 3 "comportamiento del ROI escenario base").

\subsection{Análisis de escenarios}

A continuación se describen los escenarios a evaluar:

- Sin programa de fidelización: en este escenario se ha "desactivado" el programa de fidelización para las tres categorías de clientes, de tal forma, que representa la situación de la empresa para los próximos 5 años en las condiciones actuales, asumiendo el mismo crecimiento en ventas utilizado para la prueba de ajuste.

- Cambio en los costos variables por segmento de cliente: en este escenario, se propone una modificación en los costos promedio por segmento de clientes, es decir, en aquellos costos asociados a la atención de los clientes y en promociones/ recompensas asociadas al funcionamiento del programa. Se tomaron distintos elementos de la estructura de costos por cada segmento y se generó una disminución en los costos variables unitarios. En la tabla 4 ("variables a evaluar con y sin programa de fidelización") se presenta una comparación entre el valor arrojado por Vensim para cada una de las variables que se consideran claves al momento de comparar el comportamiento del modelo ante los cambios propuestos. 
Tabla 4. Variables a evaluar Con y Sin programa de Fidelización

\begin{tabular}{|c|c|c|c|c|}
\hline Variable & $\begin{array}{c}\text { Valor Inicial } \\
\text { (Parámetros) }\end{array}$ & $\begin{array}{c}\text { Valor Final } \\
\text { Con Programa }\end{array}$ & $\begin{array}{c}\text { Valor Final } \\
\text { Sin programa }\end{array}$ & $\begin{array}{c}\text { Disminución en } \\
\text { Costos Variables }\end{array}$ \\
\hline Clientes Totales & 1.276 & 1.777 & 1.504 & 1.777 \\
\hline Clientes A & 180 & 239 & 200 & 239 \\
\hline Clientes B & 458 & 460 & 409 & 460 \\
\hline Clientes C & 638 & 1.078 & 895 & 1.078 \\
\hline CLV C A & - & $\$ 847.543,0$ & $\$ 838.063,0$ & $\$ 799.960,0$ \\
\hline CLV C B & - & $\$ 96.677,0$ & $\$ 104.588,0$ & $\$ 94.898,0$ \\
\hline CLV C B & - & $\$ 2.376,0$ & $\$ 7.505,1$ & $\$ 3.618,0$ \\
\hline Prom CLV & - & $\$ 51.799,0$ & $\$ 53.156,0$ & $\$ 49.557,0$ \\
\hline Customer Equity & - & $\$ 86.878 .300,0$ & $\$ 78.236 .500,0$ & $\$ 83.883 .600,0$ \\
\hline ROI & - & $31,20 \%$ & - & $11,98 \%$ \\
\hline
\end{tabular}

Fuente: elaboración propia a partir de resultados obtenidos

Con base en los resultados obtenidos, cabe resaltar los siguientes puntos de interés:

1. Se evidencia claramente el efecto positivo de la implementación del programa de fidelización en cuanto a número de clientes por segmento y finalmente en el número total de clientes, teniendo así un total de 1.504 clientes al final de los 5 años del periodo de análisis sin la implementación del programa, y un total de 1.777 clientes con la implementación del programa, aun en el caso en que se disminuyen los costos variables. Estos valores representan un crecimiento del 17,8 \% y del $39,3 \%$, respectivamente, en el número de clientes respecto al valor inicial (1.276 clientes). (Tabla 4 "variables a evaluar con y sin programa de fidelización).

2. La situación anterior refleja que el programa de fidelización está presentando parte de los efectos esperados como incremento en el número de clientes por la acción adicional del "efecto WOM" o "boca a boca", aunque en buena parte este incremento se debe al aumento en la cantidad de clientes $\mathrm{C}$, debido a que durante la construcción del modelo se asumió que todo nuevo cliente se considera categoría $\mathrm{C}$, puesto que no se ha establecido una relación con la empresa ni se tiene asociado un histórico de compras. A pesar de esto, este efecto de incremento en la cantidad de clientes también se evidencia en la cantidad de clientes tipos A y B.

3. En cuanto al CLV, se puede evidenciar que para los tres segmentos de clientes, se obtienen mejores resultados con la implementación del programa de fidelización 
en comparación con el escenario en el cual se disminuyen los costos; esto se debe a que al disminuir los costos, se proporciona un menor estímulo al cliente, y así, se obtiene un menor incremento en las ventas promedio por cada segmento. Sin embargo, al comparar los resultados anteriores con los resultados para el escenario sin programa de fidelización, se tiene que a excepción del CLV A, se evidencia un mayor CLV para los clientes $\mathrm{B}$ y $\mathrm{C}$, esto se debe a que en el caso de los $\mathrm{A}$, el incremento de los ingresos alcanza a justificar los costos, situación que no se presenta para los clientes B y C.

4. Combinando los efectos del incremento en la cantidad de clientes por segmento, y las variaciones descritas para el CLV, aún en el caso en el que se disminuyen los costos, la implementación del programa de fidelización representa un mejor resultado global para la empresa en la medida que el valor de su cartera de clientes es superior en un $10 \%$ respecto al valor obtenido sin la implementación del programa en el caso de estudio, y superior en un $6,7 \%$ respecto al escenario con disminución en costos promedio.

\section{CONCLUSIONES}

- En el análisis de la factibilidad de un programa de fidelización, es clave el tipo de empresa que se esté estudiando y el posterior diseño del programa, por lo cual no es posible establecer un único modelo para la evaluación de los mismos; además, se tiene una gran dependencia de las políticas y estructura financiera y de costos, asumida por la empresa que se vaya a estudiar.

- En el diseño de cualquier programa de fidelización, los tres elementos clave para su diseño son: el público objetivo al cual se orienta el programa; esto se debe a que es necesario tener en cuenta más variables como costos de atención asociados a cada cliente, o como se realizó en el caso de estudio, los factores de costo asociados a cada segmento de clientes; el segundo elemento está constituido por los canales de comunicación establecidos, ya que si se toma la decisión de invertir recursos en la implementación de una estrategia de fidelización, es más que necesario dar a conocer estas acciones a los clientes; finalmente, se tienen las recompensas asociadas a la estructura del programa de fidelización, puesto que es parte de las acciones que se desarrollan para motivar al cliente mantener y desarrollar su relación con la empresa.

- Según la estructura de funcionamiento de un programa de fidelización, con la inversión en su implementación es posible obtener un impacto positivo en el retorno sobre las inversiones que hace la empresa, aunque cabe resaltar que este no es un efecto inmediato, sino que se presenta después de un período de 
tiempo considerable en el cual se empiezan a evidenciar los beneficios asociados al programa como incremento en el número de clientes y en las ventas; y de esta manera, también se tiene un impacto sobre el estado de resultados al promover mayores ingresos para la organización.

- Para la evaluación del desempeño de un programa de fidelización, son diversas variables las que podrían considerarse; sin embargo, teniendo en cuenta las que tienen mayor impacto en el retorno sobre la inversión, vale la pena monitorizar detalladamente el comportamiento del número total de clientes en la empresa, teniendo en cuenta la composición de los mismos, es decir, la cantidad de clientes por cada segmento, el crecimiento en las ventas promedio por cliente, la estructura de costos asociada al programa, y aunque no se consideró en este modelo, es importante conocer y evaluar la satisfacción del cliente periódicamente con el fin de poder efectuar estimaciones más precisas sobre la posición de la empresa en el mercado y conocer a qué elementos se les debe prestar mayor atención para dirigir el funcionamiento y las acciones a desarrollar desde el programa de fidelización.

- El retorno sobre la inversión es un indicador financiero comúnmente aceptado y sirve como base para la toma de decisiones de la gerencia, pues permite evaluar en las mismas condiciones los resultados obtenidos tras la inversión de los recursos de la empresa en distintos proyectos.

- La dinámica de sistemas es una herramienta que permite estudiar el comportamiento de modelos complejos, donde se tiene un mayor interés sobre el comportamiento general del sistema, más que en los datos obtenidos a partir de la simulación del mismo.

\section{REFERENCIAS}

[1] K. Keller, y P. Kotler, Dirección de Marketing, México D. F.: Pearson Education, 2009.

[2] J, Córdoba, "Del marketing transaccional Marketing relacional", Entramado - Universidad Libre, Vol. 5, n. ${ }^{\circ}$, pp. 9-10, 2009.

[3] L, Valenzuela, "La gestión del valor de la cartera de clientes y su efecto en el valor global de la empresa: Diseño de un modelo explicativo como una herramienta para la toma de decisiones estratégicas de marketing”. Tesis Doctoral. Universidad Complutense de Madrid, Programa Doctoral en Ciencias Económicas y Empresariales. Madrid, España, 2007.

[4] J, Nunes, y X, Dreze, "Your loyalty program is betraying you", Harvard Business Review. Vol. 4095, pp. 3-5, 2006.

[5] J. D. Barquero, Marketing de clientes: ¿Quién se ha llevado a mi cliente?, 2. ed., Madrid, España: McGraw Hill, 2007. 
[6] Rust et al., "Return on Marketing: Using Customer Equity to focus Marketing Strategy", Journal of Marketing. Vol. 68, pp. 109-110, 2004.

[7] P, González Muñoz, "Retención vs Fidelización vs Lealtad”, Mk Marketing + Ventas, Vol. 249, pp.14-27, 2009.

[8] C, Gronroos, "The Relationship marketing process: communication, interaction, dialogue, value", Journal of Business \& Industrial Marketing, Vol.19, n. ${ }^{\circ}$ 2, pp. 99-113, 2004.

[9] M, Meyer, "White Paper on Customer loyalty metrics: A three phase Maturity Model Approach", [En Línea], Strategy to value consulting, acceso 15 de julio, 2011;Disponible:http://www. strategytovalue.com/files/Customer_Loyalty_Metrics.pdf, 2006.

[10] G. Bick, "Developing a Customer Equity model for guiding marketing spend in the financial services sector", Tesis Doctoral, University of Johannesburg, Programa Doctoral en liderazgo en desempeño y cambio. Johannesburg, Sudáfrica, 2007.

[11] B, García, “Los programas de fidelización de clientes en establecimientos detallistas: un estudio de su eficacia", Tesis Doctoral. Universidad de Valladolid, Programa Doctoral en Ciencias Económicas y Empresariales. Valladolid, España. 2009.

[12] L, Mendoza, "Critical success factors for a customer relationship management", strategy. Information and Software Technology. Vol. 49. pp. 917, 2007

[13] J. A., Martínez, y L. Martínez, "Gestión de clientes de servicios deportivos; un modelo de dinámica de sistemas", Revista Internacional de Medicina y Ciencias de la Actividad Física y el Deporte, Vol. 9, n. 36, pp. 433-434, 2009.

[14] J, Sterman, “Business dynamics: Systems thinking and modeling for a complex world”, United States of America: Mc Graw Hill, 2000. 